\title{
UNDERLYING SURFACE REMOTE SENSING BY THE MICROWAVE RADIOMETER WITH HIGH MEASUREMENT RATE
}

\author{
Anton Ubaichin ${ }^{1 *}$ Egor Alexeev ${ }^{2}$, Gregory Zhuk $^{2}$, Inna Plotnikova ${ }^{1}$, Evgeniya Timofeeva ${ }^{1}$, \\ Tilekbek Abdirasul uulu ${ }^{2}$, Daniil Danilov ${ }^{2}$ and Abdulaziz Tashhodgaev ${ }^{2}$ \\ ${ }^{1}$ Tomsk Polytechnic University, Institute of Non-Destructive Testing, 634050, Tomsk, Russia \\ ${ }^{2}$ Tomsk State University of Control Systems and Radioelectronics, 634050, Tomsk, Russia
}

\begin{abstract}
The paper describes a new approach to microwave radiometer design. The approach implies simultaneous using both modified zero measurement method and multi-receiver technique. Simultaneous using increases the operating characteristics of airborne microwave radiometers for aircrafts with self-contained power supply. The block diagram of the onboard Earth remote sensing microwave radiometric system is presented. The block diagram and operating timing diagrams of the designed radiometer are shown. An original technique to design a fiducial noise source for transfer characteristics is discussed. The advantages of the designed radiometer in comparison with the state of the art zero-type microwave radiometer are described.
\end{abstract}

\section{Introduction}

The radiometric methods are widely used in various branches of national economy. The technique level of the microwave radiometric system is the major factor of measurement accuracy, and it determines the metrological level of measurement. Unlike the mobile system, the problem of the development and design of stationary radiometric system is completely solved by means of classical measurement methods [1].

Application of microwave radiometric systems in aircrafts and other mobile objects with self-contained power is of current relevance. The main feature of the microwave radiometric system is a limited capacity of the power cell. The necessity of temperature control and power supply of the receiver gain control system leads to high energy requirements [2].

The urgency of the airborne microwave radiometric system is beyond question. One of the most important and challenging applications is monitoring of the underlying surface for search for "cold" or "hot" specific emission areas. The specific emission area can be formed as result of the fire or a hidden metallic object $[3,4]$.

\footnotetext{
*Corresponding author:ubaychin@tpu.ru
} 


\section{Theory}

We have designed a new type of the airborne microwave radiometric system for the $\mathrm{Ka}$ range. The microwave radiometric system has low energy consumption, high measurement rate and accuracy, which are inherent to the zero-type microwave radiometer.

The designed radiometric system is based on the modified zero measurement method, multi-receiver techniques and an original technical solution.

The block diagram of the radiometric system is shown in Figure 1.

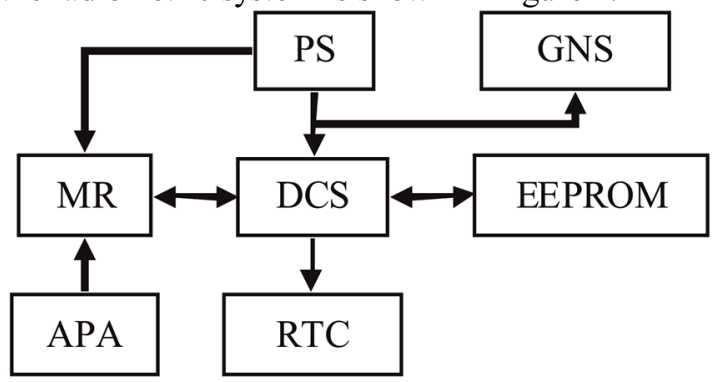

Figure 1. Block diagram of the radiomentric system.

The radiometric system consists of the antenna phased array APA, microwave radiometer MR, digital control system (DCS), electrically erasable programmable read-only memory EEPROM, radio transmitting channel RTC, self-contained power supply PS and global navigation system module GNS.

Figure 2 shows the block diagram of the two-receiver zero-type radiometer with reduced energy consumption.

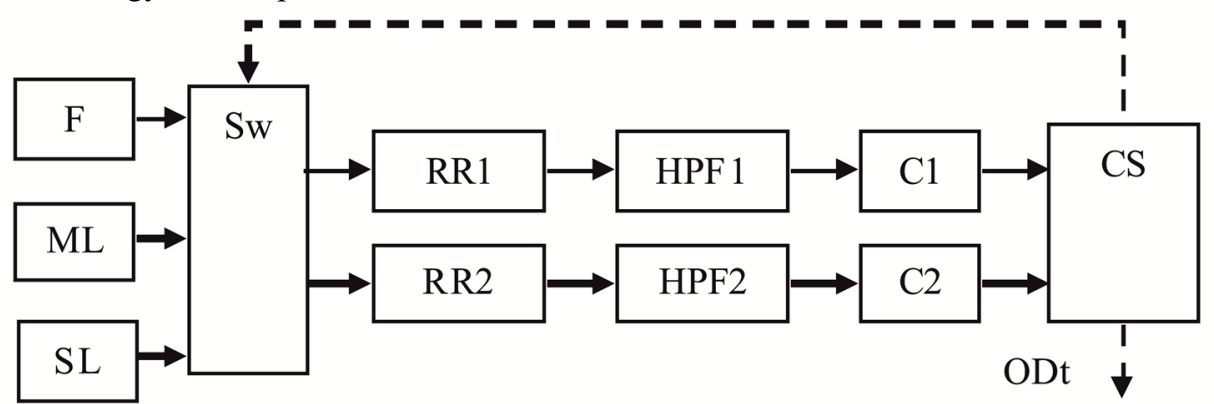

Figure 2.Designed radiometer block diagram.

The radiometer consists of antenna feeder $F$, the output of which is connected to the input of microwave switch $\mathrm{Sw}$, the corresponding outputs of which are connected to matched load ML and short load SL, herewith, the first Sw output is connected to the first input of control system CS via the first radiometric receiver RR1, high pass filter HPF1 and the first zero-crossing comparator $\mathrm{C} 1$ connected in series. The second $\mathrm{Sw}$ output is connected to the second CS input via the second radiometric receiver RR2, high pass filter HPF2 and second zero-crossing comparator C2 connected in series. The control system has a single control data bus, which connects the corresponding CS output with the control input data bus of the Sw, and the second CS data bus is the output data bus ODt of the radiometer. 


\section{Operating principle}

The microwave radiometer operates according to the timing diagrams shown in Figure 3 as follows.

a)

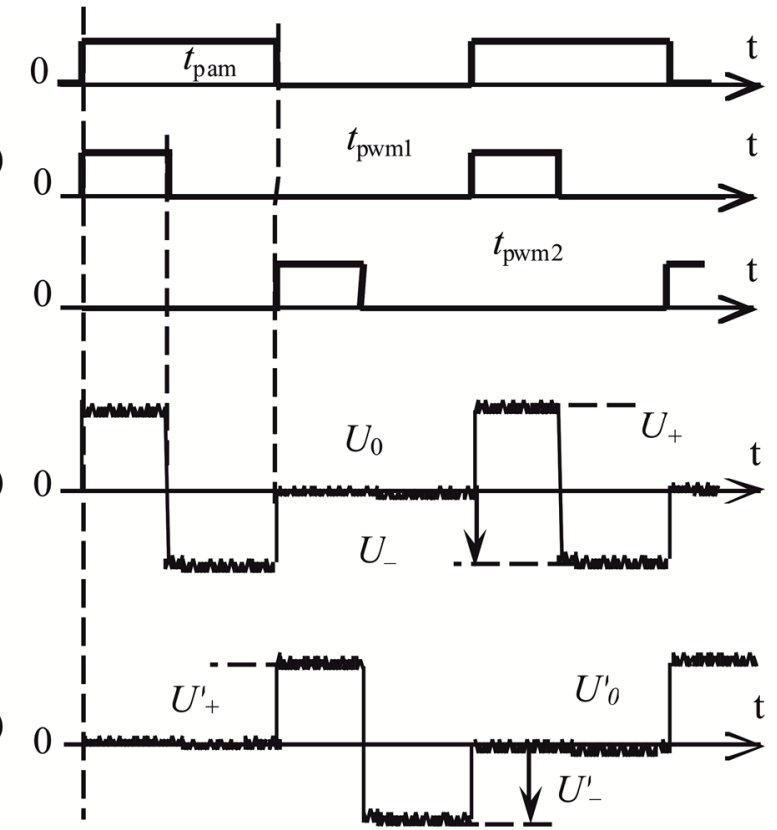

Figure 3. Operating timing diagrams of the designed radiometer.

The radiometer operates synchronously with three logical control signal $t_{\mathrm{pam}}, t_{\mathrm{pwm} 1}$ and $t_{\mathrm{pwm} 2}$ generated by CS. Sequential connection of the antenna phased array to the inputs of the first and second radiometric receivers is carried out by control signal $t_{\mathrm{pam}}$ in the microwave switch. While the $t_{\mathrm{pam}}$ is high state, the antenna phased array is connected to the input of the second receiver, low state of the $t_{\mathrm{pam}}$ corresponds to the antenna phased array connections to the input of the first receiver. The high state of control signals $t_{\mathrm{pwm} 1}$ and $t_{\mathrm{pwm} 2}$ corresponds to the matched load connections to the inputs of the first and second receivers respectively. While $t_{\mathrm{pwm} 1}$ is low and $t_{\mathrm{pam}}$ is high the short load is connected to the input of the first receiver. While both $t_{\mathrm{pwm} 2}$ and $t_{\mathrm{pam}}$ is low the short load is connected to the input of the second receiver. In Figure 3, $d$ and Figure 3, e diagrams corresponds to the voltage waveform at the input of the first and second comparators respectively.

The microwave radiometer operating algorithm is as fallows. Assume that the matched load has noise temperature $T_{\mathrm{ML}}$, the noise of first radiometric receiver is $T_{\mathrm{NR} 1}$ and the noise temperature of the antenna phased array is $T_{\mathrm{A}}$. In this case, the amplitude of positive and

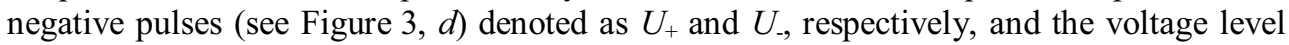
corresponding to the antenna array connection to the receiver input is $U_{0}$.

$$
\begin{aligned}
& U_{+} \approx k \Delta f_{1} G_{1}\left(T_{\mathrm{ML}}+T_{\mathrm{NR} 1}\right) \\
& U_{\approx} \approx k \Delta f_{1} G_{1}\left(T_{\mathrm{NR} 1}\right) \\
& U_{0} \approx k \Delta f_{1} G_{1}\left(T_{\mathrm{NR} 1}+T_{\mathrm{A}}\right)
\end{aligned}
$$

where $k$ is Boltzmann constant, $\Delta f_{1}$ is the bandwidth of the first radiometric receiver, $G_{1}$ is the gain of the first radiometric receiver. 
An original technique of negative pulse generation in the designed block diagram is of special interest. It implies reflection of the radiometric receiver noise in the short load. The fulfillment of condition (4) leads to the equality of the voltage-time areas [5]:

$$
\left(U_{0}-U_{0}\right)\left(t_{\mathrm{pam}}-t_{\mathrm{pwml}}\right)=\left(U_{+}-U_{0}\right) t_{\mathrm{pwm} 1}
$$

Condition (4) is fulfilled by pulse-width signal duration adjusting, while the comparator performs voltage continuous monitoring at the amplitude-modulated pulse half period corresponding to the antenna array connection to the radiometric receiver input. Determination of the zero voltage level crossings of the detected pulse series with excluded direct component in the high pass filter [3] fulfills condition (4).

Substituting expressions (1), (2) and (3) in (4) and transforming (4) relative to $T_{\mathrm{A}}$, subtract $T_{\mathrm{NR} 1}$ and dividing the left and right terms by the common multiplier $k \Delta f_{1} G_{1}$ obtain

$$
T_{\mathrm{A}=} T_{\mathrm{ML}}\left(t_{\mathrm{pwml}} / t_{\mathrm{pam}}\right)
$$

Equality (5) shows the invariance of the measurement results to the changes in both gain and noise temperature of the receiver.

\section{Experimental research}

To check the theory the experimental research series is carried out. For implement it the zero type microwave radiometer is designed in accordance with block diagram (see Figure 2). For microwave radiometer designing are used computer-aided design systems of technological documentation (Solid Works) and electromagnetic analysis (AWR Microwave Office, CST Microwave Studio). The measurement characteristics of the designed microwave modules is carried out by special equipment produced by LTD "Planar" (vector network analyzer Obzor-804/1)and JSC "Micran" (scalar network analyzer R2M-18A). Low frequency modules of the designed radiometer are tested by oscilloscope Tektronix TDS 2024B. The designed radiometer part is shown in Figure 4.

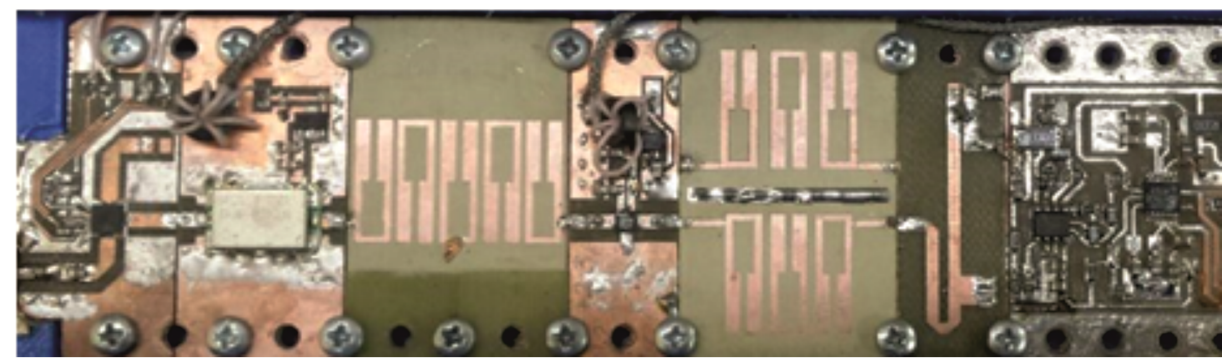

Figure 4.Designed radiometer part.

For the microwave modules designing is used foiled dielectric Flan with dielectric permittivity $\varepsilon=3.8$. This material has low cost, high technological effectiveness and satisfactorily stable of electric characteristics in compare with other dielectric material, for example ceramics. LPKF ProtoMat S100 milling machine is used for microwave printed circuit boards manufacturing.

Figure 5 shows the diagram of the low frequency part of the radiometer in the research of the negative pulse generation principle. 


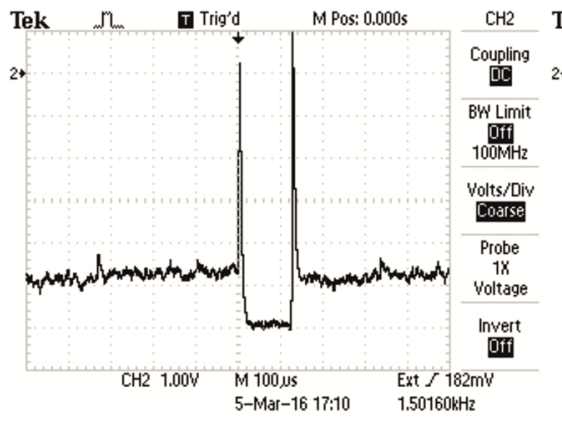

a)

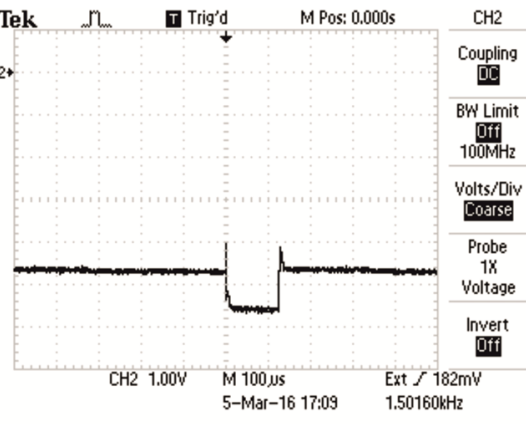

b)

Figure 5. Negative pulse generation during the microwave radiometer calibration: a) without analog averaging, b) with analog averaging.

The technique of the negative pulse generation is a one task of the research. The test technique consist in operating algorithm realization, mentioned above, when antenna equivalent connected to the system (for example, temperature controlled matched load) with noise temperature close to noise temperature of the matched load which is a part of the microwave radiometer. Wherein, the output diagrams of the low frequency part of the radiometer should have only negative pulse, which is proportional to the system noise temperature.

The high state of the signal in Figure 5 corresponds to the fiducial connection to the system input (matched load and antenna equivalent) with noise temperature close to $300 \mathrm{~K}$, low state corresponds to system input connection to the microwave short load during the time interval $t_{\mathrm{PWM}}$. Analog averaging is performed by low pass filters with $\mathrm{RC}$ time $\tau=24 \mathrm{~ms}$.

Figure 6 shows the time diagram of zero balance achieving in the system. Wherein, at the $t_{\mathrm{PWM} 2}$ time interval, fiducial with noise temperature $T_{\text {Nlow }}=150 \mathrm{~K}$ is connected to the system input.

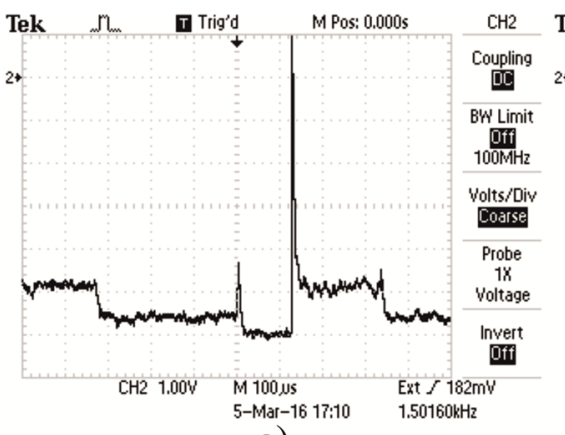

a)

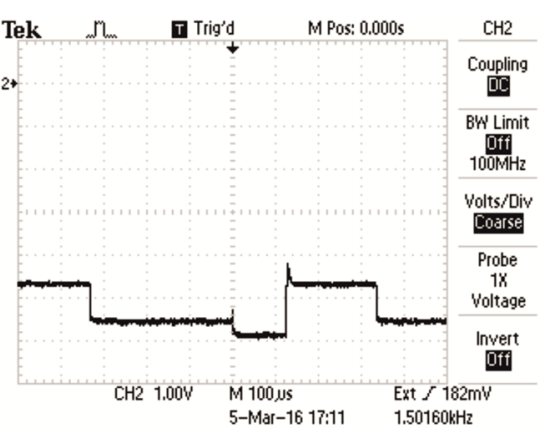

b)

Figure 6.Time diagram of zero balance achieving in the system: a) without analog averaging, b)with analog averaging.

The high state of the signal corresponds to connection of the matched load with noise temperature $T_{\mathrm{ML}}=300 \mathrm{~K}$ to the system input, middle level corresponds to the antenna equivalent signal with noise temperature $T_{\mathrm{N} \text { low }}=150 \mathrm{~K}$, low state corresponds to signal, that proportional to system noise when microwave short is connected to the input. Analog averaging performed similar with diagram at Figure 5. 


\section{Discussion}

The analysis of the diagrams of the low frequency part of radiometric system (see Figure 5 and Figure 6) and compare with theory (Figure 3) shows the full operability of the designed method. The technique to negative pulse generation make possible to realize the zero type measurement without using additional active noise fiducial sources.

The researches of designed system shown the system dynamic range is $0 \ldots 300 \mathrm{~K}$, wherein, the upper limit determined by temperature of the matched load which is a part of the system. System noise is about $75 \mathrm{~K}$, which is satisfactorily with considering of applied technology and electronic components.

Interesting results were obtained by analysis of the diagram without averaging. There are specific voltage spikes when noise references are switching. The analysis of the spikes and their characteristics leads to conclusion about the need to changing a synchronization technique of low pass filters and microwave switch which in the system input, more thorough screening of the analog measuring parts or using a digital filtering.

\section{Conclusion}

A new approach to design the microwave radiometer by the zero measurement method and multi-receiver technique is presented. The designed two-receiver zero-type microwave radiometer uses the receiver noise temperature and matched load as fiducial points for transfer characteristics. This feature allows us to avoid the use of the fiducial noise generators based on the impact avalanche and transit-time diode and Gunn diode. In comparison with the radiometer described in [5], the designed radiometer has a simplified structure as it excludes the directional coupler, noise generator and second microwave switch. The described radiometer does not need the matched load temperature control for measurement result correction.

The generation of the fiducial noise signal by the reflection of the receiver noise is associated with a number of the features described, for example, in [5]. In the considered radiometer, these features can also be observed, including those associated with the receiver noise interference. The method to eliminate these features is protected by "know-how".

In the designed radiometer microwave switch based on the Peregrine Semiconductor PE4257 microchip, it make possible to realize mode of alternately connection of the antenna, microwave short load and matched load to the receiver input. Low noise amplifiers realized by Minicirquits PMA5453+ microchip. The Schottkydiode HSMS-2850 together with matched circuit is used for detection of microwave signals. Low frequency modules based on the Analog Devices microchips. Digital control system is realized by AT91SAM7S256 microchip.

For microwave radiometer designing we are used electronic components of foreign manufacturing. This fact is significant disadvantage of this new technical solution. In spite of this, designing concept implies using only domestic electronic components in the case of serial production of the designed system after successfully passed of field tests series.

Besides of a microwave measuring parts, the designing on board system is supplemented by new sensors to improve in formativeness of remote sensing. These include the infrared cameras. Operating principle, design technique and integration to the measurement system will take place in the near future.

In future we want to carry out field and laboratory tests. Laboratory tests including the researches of stability and fluctuation sensitivity, field tests means the researches of Siberian Federal District areas to estimation of the designing system efficiency during the different types of underlying surface researching. 


\section{Acknowledgments}

The reported study was partially supported by RFBR, research project No. 16-37-00237 mol_a.

\section{References}

[1] A. Camps, J.Tarongi, Rem. Sens. 2, 191(2010) doi:10.3390/rs2010191

[2] A. Harvey, R. Appleby, Aeron. J. 107, 87 (2003)

[3] Y. Li , H. Zhao, J. Fan, MATEC Web of Conferences 22, 04008 (2015) doi: 10.1051/matecconf $/ 20152204008$

[4] A. Bespalko, A. Surzhikov, L.Yavorovich, P. Fedotov, Russ. J. Nondestr. Test. 48, 221 (2012) doi: 10.1134/S1061830912040043

[5] A.V. Filatov, A.V. Ubaichin, D.E. Paraev, Inst. and Exp. Tech. 55, 59 (2012) doi: $10.1134 / \mathrm{S} 0020441211060066$ 